\title{
Atomic structure considerations for the low-temperature opacity of Sn
}

\author{
J. Colgan ${ }^{1}$, D. P. Kilcrease ${ }^{1}$, J. Abdallah, Jr. ${ }^{1}$, M. E. Sherrill ${ }^{1}$, \\ C. J. Fontes ${ }^{1}$, P. Hakel ${ }^{1}$, and G. S. J. Armstrong ${ }^{1,2}$ \\ ${ }^{1}$ Los Alamos National Laboratory, Los Alamos, NM 87545 \\ ${ }^{2}$ J. R. Macdonald Laboratory, Kansas State University, Manhattan, KS 66506
}

\begin{abstract}
We have begun a preliminary investigation into the opacity of Sn at low temperatures $(<50 \mathrm{eV})$. The emissivity and opacity of Sn is a crucial factor in determining the utility of Sn in EUV lithography, with numerous industrial implications. To this end, we have been exploring the accuracy of some approximations used in opacity models for the relevant ion stages of $\mathrm{Sn}$ (neutral through $\sim 18$ times ionized). We find that the use of intermediate-coupling, as compared to full configuration-interaction, is not adequate to obtain accurate line positions of the important bound-bound transitions in Sn. One requires full configuration-interaction to properly describe the strong mixing between the various $n=4$ sub-shells that give rise to the $\Delta n=0$ transitions that dominate the opacity spectrum at low temperatures.

Since calculations that include full configuration-interaction for large numbers of configurations quickly become computationally prohibitive, we have explored hybrid calculations, in which full configuration-interaction is retained for the most important transitions, while intermediate-coupling is employed for all other transitions. After extensive exploration of the atomic structure properties, local-thermodynamicequilibrium (LTE) opacities are generated using the ATOMIC code at selected temperatures and densities.
\end{abstract}




\section{Introduction}

The radiative properties of high-Z plasmas are important for a number of applications, including industrial processes that focus on the development of extreme ultraviolet (EUV) lithography. The main focus of such studies is the development of an efficient radiation source near $13.5 \mathrm{~nm}$ that has high power and narrow bandwidth, which can allow the production of processors with ever smaller components for the semiconductor industry [1]. The radiative properties of Sn have long been known to largely meet these requirements. However, the development of predictive models of the emissivity and opacity of Sn at the temperatures of relevance (around 20-40 eV) has been more difficult, for the most part due to the complexity of the ion stages of relevance (nominally around $\mathrm{Sn}^{5+}$ to $\mathrm{Sn}^{15+}$ ). However, important studies have been made into the atomic structure properties of such ion stages [1-7], and also the opacity of Sn at these conditions $[8,9]$. The radiative emission is of course the quantity of most interest, but the opacity of Sn remains important because light emitted from a Sn plasma may be strongly absorbed during its propagation through the plasma. In 2005, one of the first measurements was made of the opacity of Sn at the conditions of most interest to lithography [10]. The measured transmission was compared to HULLAC calculations and reasonable agreement was found, although such agreement required the postulation of a higher plasma temperature than that predicted from hydro simulations. A later study [8] found improved agreement with the measured transmission, although discrepancies remained. Unfortunately, few other studies exist of the opacity of midto high-Z materials. This is in part because many of the major worldwide opacity efforts (see, for example, [11-15]) have focused on elements with Z $<30$, since these efforts were primarily carried out for astrophysical research in stellar modeling, for which most of the interest is in elements from hydrogen through nickel. Of course, another reason is the sheer complexity of the ion stages of interest; most relevant $\mathrm{Sn}$ ion stages have open $4 d$ subshells, and mixing within the $n=4$ manifold between the many levels that exist produce large numbers of transitions and is computationally difficult to treat.

In this paper we report on our efforts to compute the opacity of Sn plasmas at temperatures from around 20-40 eV. We use the Los Alamos suite of atomic physics codes (for an overview, see [16]) and specifically the CATS code $[17,18]$ to generate atomic structure models that contain fine-structure energy levels and the corresponding transition probabilities. The multi-purpose ATOMIC code $[19,20]$ is then used to compute the opacity of Sn assuming local thermodynamic equilibrium (LTE) conditions. ATOMIC has recently been used to produce comprehensive opacity tables for hydrogen through zinc [21]. These tables are publicly available [22] and have already been used in several stellar modeling efforts [21,23]. 
In the next section we briefly discuss the methods used in our calculation, and describe the configurations chosen for the Sn ion stages of relevance. We then present Sn opacities at a number of temperatures of interest, and compare to the transmission measurements of [10]. We end with a short discussion of our ongoing work and future plans.

\section{Calculation details}

The major aspects of how opacities are computed within ATOMIC have been previously discussed by us $[21,24,25]$. The Los Alamos semi-relativistic atomic structure code CATS [18] (based on Cowan's codes [17]) is used to compute atomic structure properties, primarily energy levels and transition probabilities. The GIPPER code [26] provides photoionization cross sections. These codes compute data that are read into the ATOMIC code [19,20] and used, along with the equation-of-state (EOS) computed using the same dataset through our ChemEOS model [27], to produce opacities for user-specified temperatures and densities.

In this study, we focus on the nature of the CATS atomic structure calculations, using a relatively small set of configurations for each Sn ion stage. This set of configurations is almost certainly insufficient to produce converged atomic datasets that can be used to compute opacities over wider temperature and density ranges. Our primary aim in this study is instead to determine how accurate the atomic data needs to be, in order to obtain an accurate opacity. Specifically, we present calculations in which full configuration-interaction (CI) is used to generate the fine-structure energy levels from the input set of configurations. Such calculations include interactions between the configurations and are expected to be accurate for moderately-charged ions, as in this study. The basis of our approach is the Hartree-Fock method as implemented in the Cowan codes and discussed in detail by [17], where examples are provided of the increase in accuracy of the computed energy levels as the charge of the ion increases [28]. The same set of configurations is also used to generate fine-structure levels using intermediate-coupling (IC), that is, only retaining interactions between levels arising from the same configuration. The IC calculations are significantly less computationally expensive than the CI calculations. We note that the CI calculations described here take long times and large RAM resources to complete on the machines available to us, and significantly larger CI calculations would be difficult, if not impossible, to complete at present. We also note that atomic structure calculations based on the IC approximation were used in the generation of our recently released opacity tables [21], which was necessary due to the large number of configurations that had to be included in those calculations. One of the main aims of this present study is to determine whether atomic structure calculations that retain only 
IC are sufficient for Sn opacities. This is important to understand, since it seems unlikely that full-CI calculations can be utilized in the foreseeable future for calculations that include the large numbers of configurations that are necessary for obtaining converged opacity tables.

The choice of configurations included in our models was based on several considerations. We consider which configurations were relevant in terms of producing important transitions for the ion stages under consideration, and also which configurations were important in terms of CI mixing for such configurations. These considerations were then balanced by what calculations are actually possible (in a computational runtime and RAM sense). In these considerations, we were able to draw on several useful previous studies $[1,8,10]$. The model that we use in the work described here typically has one-, two- and three-electron excitations from the valence subshell of the ground configuration of each ion stage into available $n=4$ subshells. We also include single and double excitations from the inner $4 s$ and $4 p$ subshells of the ground configuration. We allow single excitation from the $n=4$ subshells into configurations with $n=5$ through $n=8$. Finally, two-electron excitations into the $n=5$ subshells (normally from the valence subshell of the ground configuration) are also retained. As an explicit example, we list below the configurations retained for $\mathrm{Sn}^{10+}$, where we assume in all cases a filled Ni-like core. We included the configurations $4 s^{2} 4 p^{6} 4 d^{4}$, $4 s^{2} 4 p^{6} 4 d^{3} 4 f, 4 s^{2} 4 p^{6} 4 d^{2} 4 f^{2}, 4 s^{2} 4 p^{6} 4 d^{1} 4 f^{3}, 4 s^{2} 4 p^{6} 4 f^{4}, 4 s^{2} 4 p^{5} 4 d^{5}, 4 s^{2} 4 p^{5} 4 d^{4} 4 f$, $4 s^{2} 4 p^{5} 4 d^{3} 4 f^{2}, 4 s^{1} 4 p^{6} 4 d^{5}, 4 s^{1} 4 p^{6} 4 d^{4} 4 f, 4 s^{1} 4 p^{6} 4 d^{3} 4 f^{2}, 4 s^{2} 4 p^{4} 4 d^{6}, 4 s^{2} 4 p^{4} 4 d^{5} 4 f$, $4 s^{2} 4 p^{6} 4 d^{3} n l, 4 s^{2} 4 p^{6} 4 d^{2} 4 f n l, 4 s^{2} 4 p^{5} 4 d^{4} n l, 4 s^{2} 4 p^{5} 4 d^{3} 4 f n l, 4 s^{1} 4 p^{6} 4 d^{4} n l, 4 s^{1} 4 p^{6} 4 d^{3} 4 f n l$, $4 s^{2} 4 p^{4} 4 d^{5} n l, 4 s^{2} 4 p^{6} 4 d^{2}(5 l)^{2}$, and $4 s^{2} 4 p^{5} 4 d^{3} 5 s 5 l$. Here, $n$ ranges from 5 to 8 and $l \leq 3$. Similar sets of configurations were retained for the neighboring ion stages. Full CI calculations, as well as IC calculations, were performed for the ion stages $\mathrm{Sn}^{10+}$ through $\mathrm{Sn}^{17+}$. We note that the list of configurations discussed here is not necessarily complete and that other configurations may produce additional transitions that can contribute to the Sn opacity in the regions considered in this work. We further note that more configurations may be necessary to ensure a converged partition function, which is important in obtaining the correct level populations. This is the topic of ongoing work, and we restrict ourselves in this study to investigations of the importance of the accuracy of the atomic energy levels.

In figure 1 we present the opacity of $\mathrm{Sn}$ at a temperature of $40 \mathrm{eV}$ and mass density of $0.01 \mathrm{~g} / \mathrm{cm}^{3}$, conditions similar to those thought to be present in recent measurements [10]. These calculations include the ion stages $\mathrm{Sn}^{10+}$ through $\mathrm{Sn}^{17+}$. We compare the full CI calculations (black line) with IC calculations (red line). We immediately notice that the opacity from the IC calculation is markedly different from the CI calculation, with significant redistribution of oscillator strength in the IC calculation away from the intense, narrow feature found in the CI calculation. The CI calculations performed here required significant computational resources for some of the lower-charged ion 


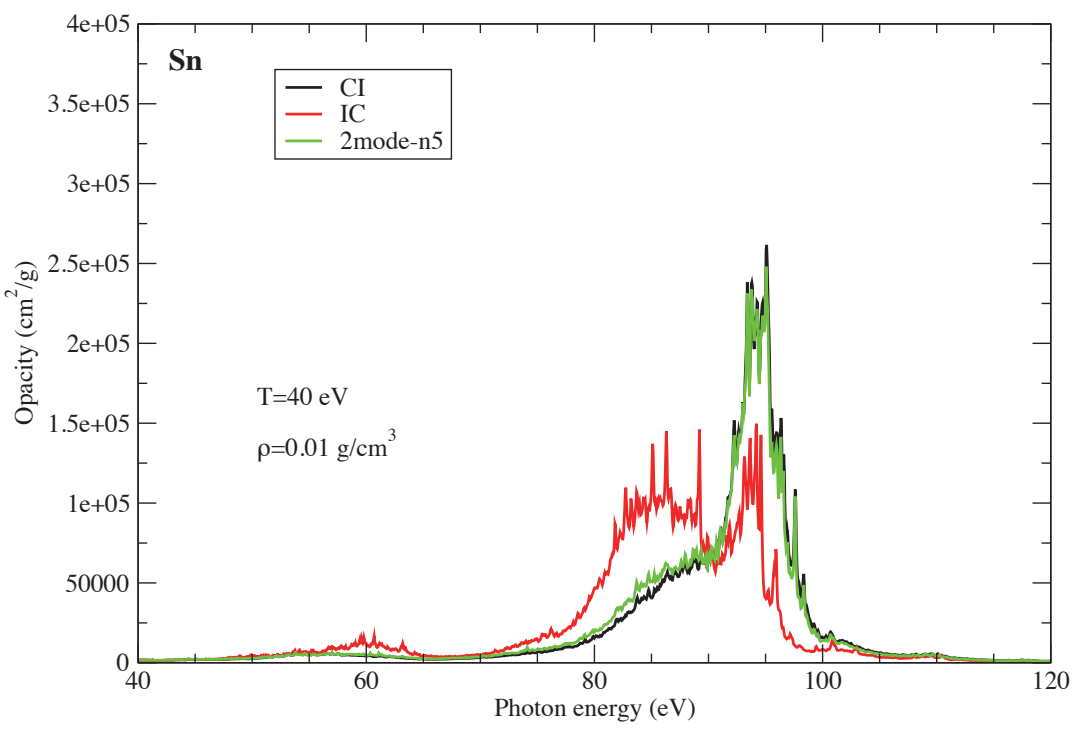

Fig. 1. Opacity of Sn at a temperature of $40 \mathrm{eV}$ and at a mass density of $0.01 \mathrm{~g} / \mathrm{cm}^{3}$. We compare ATOMIC calculations using three different atomic structure models. The black line uses atomic structure calculations that include full configuration-interaction (CI) as described in the text and the red line uses the same structure model, but computed using only intermediate-coupling (IC). The green line shows a '2-mode' calculation, in which CI is retained for all transitions involving $\Delta n=0$ and $\Delta n=1$ transitions from the $n=4$ manifold. See text for details.

stages, and we found that extending such CI calculations to even lower charged ion stages was not possible at this time. In an effort to produce substantially equivalent atomic structure models, but at a lower computational cost, we then modified our code to retain only CI among configurations that produce $\Delta n=0$ and $\Delta n=1$ transitions from the $n=4$ manifold. The remaining transitions $(\Delta n=2$, etc), are computed within the IC approximation. This calculation, labeled as '2mode-n5' in figure 1 is shown as the green line, and is found to be very similar to the full CI calculation. Subsequently, this approach allows us to extend our model (described in the previous paragraph) to all ion stages of $\mathrm{Sn}$ from neutral through $\mathrm{Sn}^{17+}$.

\section{Results}

We now discuss several convergence aspects of the opacity of $\mathrm{Sn}$ at various temperatures and at a density of $0.01 \mathrm{~g} / \mathrm{cm}^{3}$. In figure 2 we present the opacity of $\mathrm{Sn}$ at four temperatures as indicated. These temperatures span the range over which the measurement of [10] was performed, and encompass the tem- 

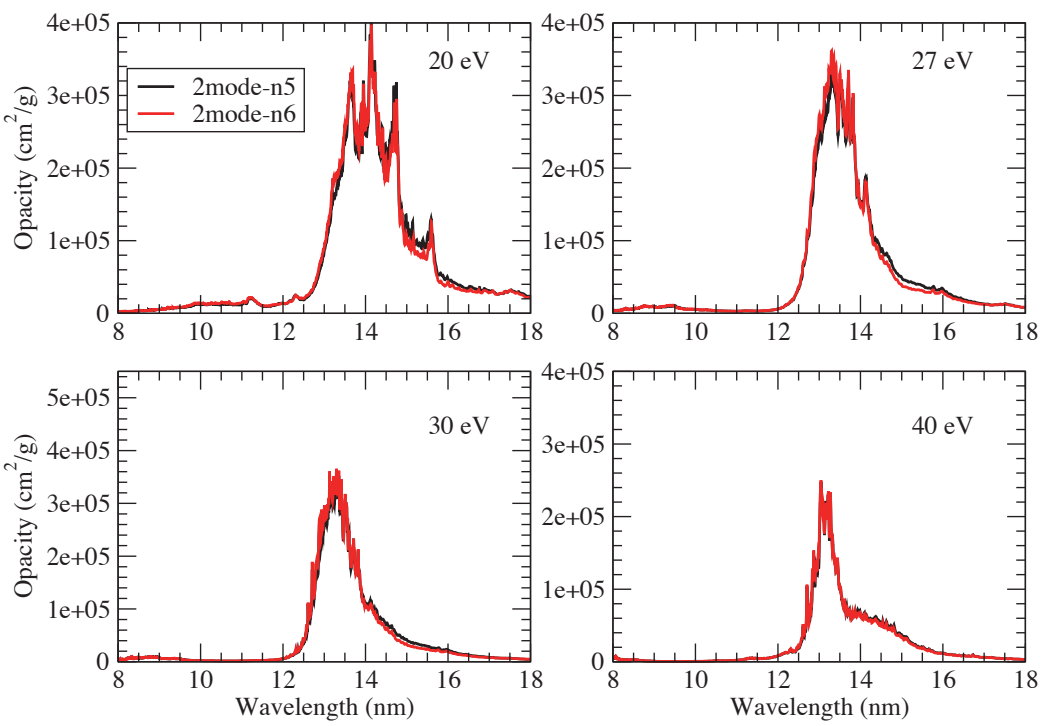

Fig. 2. Opacity of Sn at various temperatures as indicated and at a mass density of $0.01 \mathrm{~g} / \mathrm{cm}^{3}$. We compare ATOMIC calculations made using 2mode-n5 and 2mode-n6 models. See text for details.

peratures of most interest for EUV lithography applications. We find, as in a number of previous studies $[1,8,10]$ that the opacity of Sn exhibits an intense, quite narrow feature around $13.5 \mathrm{~nm}$. Although we only present the opacity here, the (LTE) emissivity has an almost identical shape over this wavelength range. The 2 mode-n5 calculations presented in figure 2 (black lines) are compared with 2 mode-n6 calculations (red lines), which are made with the same set of configurations, but now include all configurations that produce $\Delta n=2$ transitions in the full CI portion of the calculation. The two sets of calculations are very similar, indicating that CI contributions involving configurations that include $n=6$ subshells are quite minor.

In figure 3 we present calculations made with an 'extended' 2mode-n 5 model for the same temperatures and density as in figure 2. This model (labeled 2mode-n5-e) included very similar sets of configurations as the 2mode-n5 model, except that the range of angular momentum included was extended from $l \leq 3$ to $l \leq 4$, so that configurations that contain the $5 g$ orbital are included in the full CI portion of the calculation, and transitions involving configurations that contain the $6 g-8 g$ orbitals are included in the IC part of the calculation. We also included 3-electron excitations from the $4 p$ subshell for the $\mathrm{Sn}^{7+}$ and more highly charged ions. We find that, although the main opacity features between the two calculations are similar, inclusion of these states modifies the dominant opacity peak, which becomes somewhat narrower and more intense for most of the temperatures considered. This indicates that 

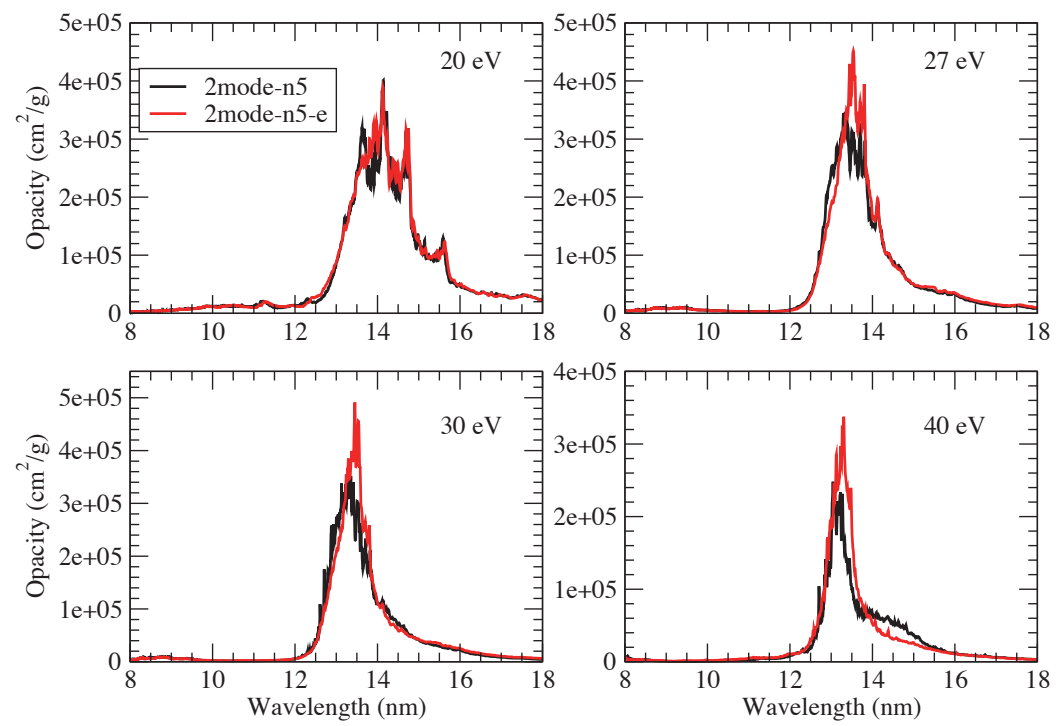

Fig. 3. Opacity of $\mathrm{Sn}$ at various temperatures as indicated and at a mass density of $0.01 \mathrm{~g} / \mathrm{cm}^{3}$. We compare ATOMIC calculations made using the 2mode-n 5 and extended 2mode-n5-e models. See text for details.

converging the opacity (or emission) of Sn at these conditions can be a difficult and non-trivial task. We also note that our calculation at $27 \mathrm{eV}$ and density of $0.01 \mathrm{~g} / \mathrm{cm}^{3}$ (upper right panel of figure 3) appears to be in quite good agreement with the opacity calculations of Zeng et al. [8].

In figure 4 we finally turn to comparisons of our ATOMIC calculations (made using the '2mode-n5-e' model described previously) with the transmission measurements of [10]. The paper of Fujioka et al. [10] was accompanied by $1 \mathrm{D}$ radiation-hydrodynamic simulations that indicated a plasma temperature of around $30 \mathrm{eV}$ and a mass density of $0.01 \mathrm{~g} / \mathrm{cm}^{3}$. The study also included HULLAC calculations. The HULLAC calculations at $30 \mathrm{eV}$ were only moderately in agreement with the measured transmission, predicting a much broader transmission dip that was at somewhat higher wavelengths. However, a HULLAC calculation at a temperature of $40 \mathrm{eV}$ was in reasonable agreement with the measured transmission. The calculations of Zeng et al. [8] at $30 \mathrm{eV}$ were in reasonable agreement with the measured transmission although some significant discrepancies remained.

The ATOMIC calculations at a temperature of $30 \mathrm{eV}$ and density of $0.01 \mathrm{~g} / \mathrm{cm}^{3}$ are shown as the red line in figure 4 . We find reasonable agreement with the measured transmission. The calculations exhibit a much steeper decrease in transmission at around $12 \mathrm{~nm}$ than the measurement, and predicts a slightly lower wavelength for the position of the maximum transmission dip (around 


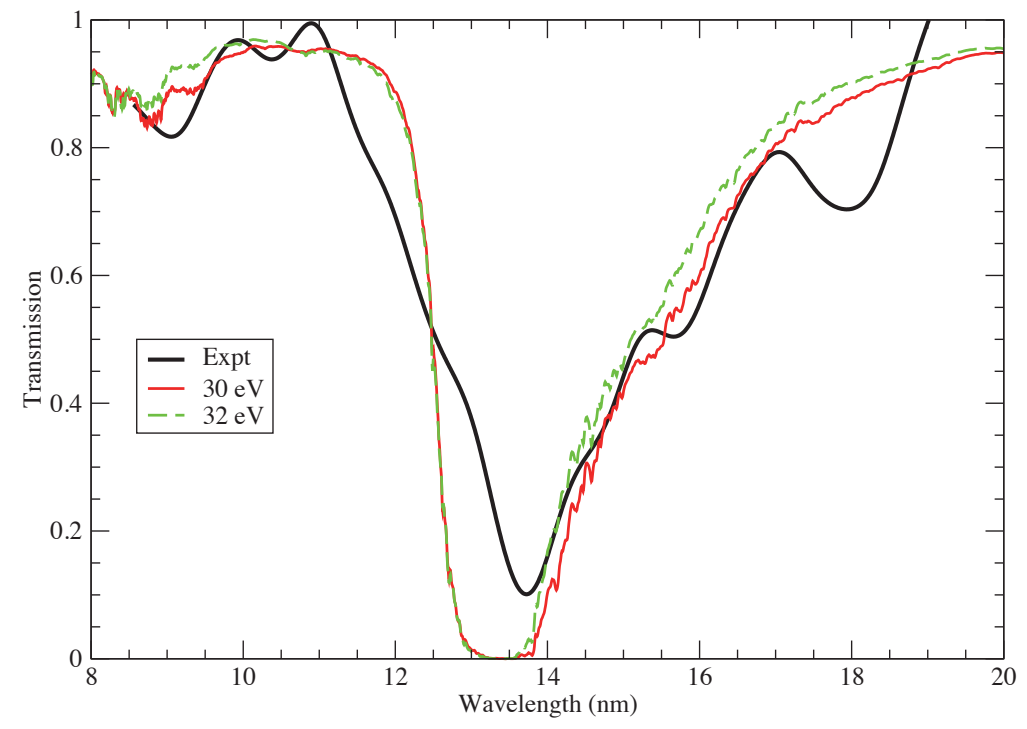

Fig. 4. Comparison of the measured transmission of Sn from [10]. We compare the experimental data with ATOMIC calculations (made using the 2mode-n5-e model) at various temperatures as indicated. To determine the transmission, a path length of $2.04 \times 10^{-3} \mathrm{~cm}$ was used [10].

$13.3 \mathrm{~nm}$ in the calculation compared to around $13.6 \mathrm{~nm}$ in the measurement). We note in passing that the measured data are smoothed, due to the resolution of the spectrometer used in the experiment. At larger wavelengths (above $14 \mathrm{~nm}$ ) the ATOMIC calculations at $30 \mathrm{eV}$ are in good agreement with the measurement. We note that this was a region in which previous calculations $[8,10]$ were not in good agreement with the measured data. An ATOMIC calculation is also presented at $32 \mathrm{eV}$, and we find that the slight increase in temperature produces a transmission at a similar level of agreement with the measured data above $14 \mathrm{~nm}$, but shows almost no difference in the level of agreement below $\sim 13.5 \mathrm{~nm}$.

\subsection{Atomic structure considerations for other elements}

We now consider if the findings in this study have any major implications regarding our previous opacity efforts [21]. In particular, noting that the majority of atomic structure calculations made for the opacity tables for $\mathrm{H}$ through $\mathrm{Zn}$ were made using the IC approximation (often through the further MUTA approximation with regard to ion populations $[21,24]$ ), it is worthwhile assessing the accuracy of such calculations in light of the large differences observed between the IC and CI calculations shown here for Sn. 

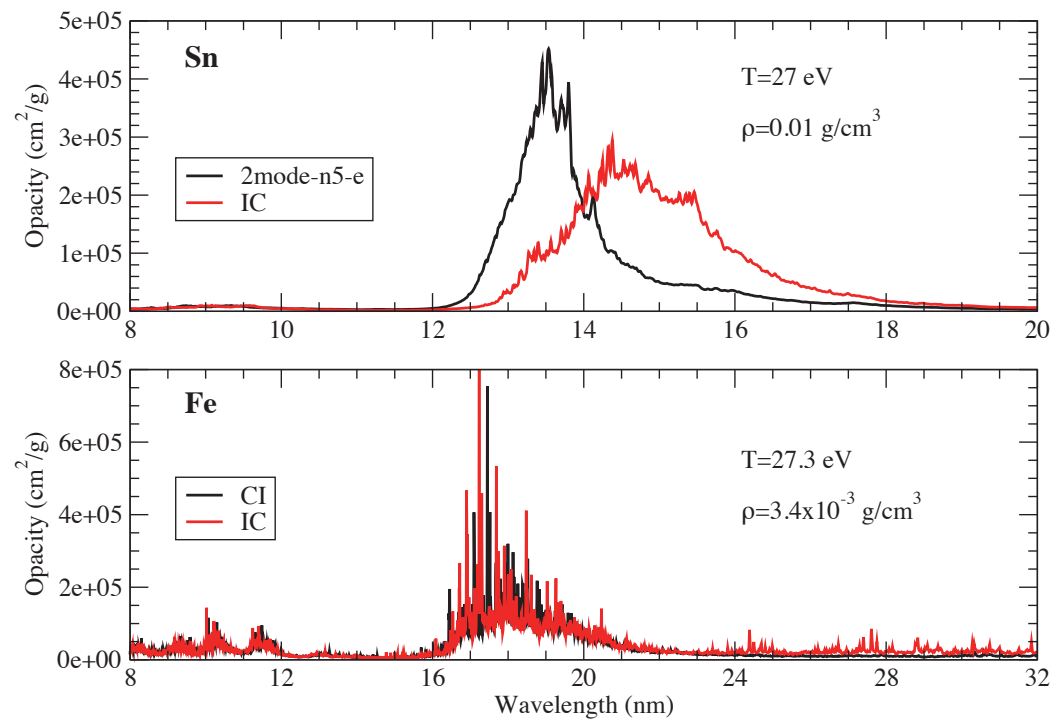

Fig. 5. Opacity of Sn (upper panel) and Fe (lower panel) opacities as a function of wavelength and at a temperature of $27 \mathrm{eV}$. For the Sn case, we compare 2 mode-n5-e calculations with intermediate-coupling (IC) calculations. For the Fe case, we compare configuration-interaction (CI) calculations with IC calculations. See text for details.

In figure 5 we show again in the upper panel the opacity of $\mathrm{Sn}$ at $27 \mathrm{eV}$ and $0.01 \mathrm{~g} / \mathrm{cm}^{3}$, and compare the IC calculations (red line) with a 2mode-n 5 calculation, in which CI is included among all configurations that contribute to $\Delta n=0$ and $\Delta n=1$ transitions. Again, we note the significant redistribution in oscillator strength in the IC calculation and the shift (and broadening) of the major opacity feature towards higher wavelengths. The lower panel of figure 5 shows the opacity of $\mathrm{Fe}$ at a similar temperature and at a slightly lower density $\left(3.4 \times 10^{-3} \mathrm{~g} / \mathrm{cm}^{3}\right)$. We compare full CI calculations with IC calculations made using a restricted 'n5'-model that was discussed recently [29]. In contrast to the Sn case, we find that the Fe opacity produced from the $\mathrm{CI}$ and IC calculations are quite similar. The position of the prominent boundbound feature is at almost the same wavelength in both calculations, unlike the $\mathrm{Sn}$ case. There is some difference in the opacity at higher wavelengths (around $25 \mathrm{~nm}$, or $50 \mathrm{eV}$ ), where the CI calculation is significantly smaller than the IC opacity calculation. This difference is partly responsible for a difference in about $5 \%$ between the Rosseland mean opacities computed from the CI and IC calculations [29].

We thus find that the significant difference caused by the inclusion of CI in Sn opacity calculations compared to IC calculations is not observed at similar conditions in a lower Z element-Fe (although, of course, it is hard to be 
definitive since we have not probed the opacity over wide ranges of temperature and/or density). We note that in the Fe calculations presented here, we are probing ion stages of a similar charge to the ion stages of relevance to the Sn calculations - roughly ions that are 10 times ionized. For Fe, this implies that the important ion stages are those with ground configurations with open $3 p$ sub-shells, with the most relevant opacity contributions involving $3 p-3 d$ transitions. For Sn, as already noted, the important transitions are chiefly $4 d-$ If transitions, from ion stages that have an open $4 d$ subshell as the ground configuration. It appears therefore that CI is relatively more important in $\Delta n=0$ transitions involving subshells with larger units of angular momentum (in the Sn case) compared to transitions between subshells with smaller units of angular momentum. This underscores the difficulty and complexity of accurate calculations of radiative properties of mid-Z elements such as Sn.

\section{Conclusion}

In future work we plan to continue our studies of Sn opacities (and emissivities). Several important issues remain. Although we have shown that our CI calculations are in reasonably good agreement with transmission measurements [10], it is hard to be certain that we have sufficient CI effects included in our models without further time-consuming calculations. However, such calculations are underway. Also, for the production of more comprehensive opacity tables (that cover wider density, temperature and photon energy ranges), one must consider larger numbers of configurations in each ion stage to ensure a converged partition function. We must also include configurations that represent inner-shell excitations to properly compute the opacity at higher photon energies. Such transitions will almost certainly have to be computed using IC approximations, and we are building towards such calculations by assessing how many configurations are required for convergence over a range of densities. Finally, we note that studies indicate [30] that the conversion efficiency of Sn as an intense emission source is increased at lower ion densities. In such situations the plasmas are much more likely to be in non-LTE. This can greatly complicate theoretical calculations, since one needs to explicitly include rates for electron-driven processes and solve the collisional-radiative (CR) equations to determine ion populations, instead of using LTE approximations such as the Saha-Boltzmann relations. For example, an analysis of large-scale CR modeling with varying degrees of CI has been recently provided for an illustrative case [31]. As discussed in [31], explicit calculations of all the excitation and ionization cross sections for the large numbers of fine-structure levels considered in the current LTE calculations could be very computationally expensive, along with obtaining a solution to the resulting rate equations. 


\section{Acknowledgments}

The Los Alamos National Laboratory is operated by Los Alamos National Security, LLC for the National Nuclear Security Administration of the U.S. Department of Energy under Contract No. DE-AC52-06NA25396.

\section{References}

[1] A. Sasaki, A. Sunahara, H. Furukawa, K. Nishihara, S. Fujioka, T. Nishikawa, F. Koike, H. Ohashi, and H Tanuma, J. Appl. Phys. 107, 113303 (2010).

[2] A. Windberger et al., Phys. Rev. A 94, 012506 (2016).

[3] R. D'Arcy et al., J. Phys. B 42, 165207 (2009).

[4] R. D'Arcy et al., Phys. Rev. A 79, 042509 (2009).

[5] S. S. Churilov and A. N. Ryabtsev, Optics and Spectrosc. 100, 660 (2006).

[6] J. White, P. Hayden, P. Dunne, A. Cumings, N. Murphy, P. Sheridan, and G. O'Sullivan, J. Appl. Phys. 98, 113301 (2005).

[7] H. Ohashi, S. Suda, H. Tanuma, S. Fujioka, H. Nishimura, A. Sasaki, and K. Nishihara, J. Phys. B 43, 065204 (2010).

[8] J. Zeng, C. Gao, and J. Yuan, Phys Rev. E 82, 026409 (2010).

[9] M. Lysaght et al., Phys. Rev. A 72, 014502 (2005).

[10] S. Fujioka et al., Phys. Rev. Letts. 95, 235004 (2005).

[11] M. J. Seaton, Y. Yan, D. Mihalas, and A. K. Pradhan, Mon. Not. R. Astron. Soc. 266, 805 (1994).

[12] N. R. Badnell et al., Mon. Not. R. Astron. Soc. 360, 458 (2005).

[13] F. J. Rogers and C. A. Iglesias, Astrophys. J. S 79, 507 (1992).

[14] N. H. Magee et al., Astronom. Soc. Pacific Conf. Series 78, 51 (1995).

[15] C. Blancard, P. Cossé, and G. Faussurier, Astrophys. J. 745, 10 (2012).

[16] C. J. Fontes, H. L. Zhang, J. Abdallah Jr, R. E. H. Clark, D. P. Kilcrease, J. Colgan, R. T. Cunningham, P. Hakel, N. H. Magee and M. E. Sherrill, J. Phys. B 48, 144014 (2015).

[17] R. D. Cowan, The Theory of Atomic Structure and Spectra (University of California Press, Berkeley, 1981).

[18] J. Abdallah, Jr., R. E. H. Clark and R. D. Cowan, Los Alamos Manual LA11436-M, Vol. I, 1988. 
[19] N. H. Magee et al., 14th Topical Conference on Atomic Processes in Plasmas, Eds: J. S. Cohen, S. Mazevet, and D. P. Kilcrease, (New York: AIP), pp 168-179 (2004).

[20] P. Hakel et al., J. Quant. Spectrosc. Rad. Trans. 99, 265 (2006).

[21] J. Colgan, D. P. Kilcrease, N. H. Magee, M. E. Sherrill, J. Abdallah, Jr., P. Hakel, C. J. Fontes, J. A. Guzik, and K. A. Mussack, Astrophys. J. 817, 116 (2016).

[22] http://aphysics2.lanl.gov/opacity/lanl

[23] P. Walczak, C. J. Fontes, J. Colgan, D. P. Kilcrease, and J. A. Guzik, Astron. \& Astrophys. 580, L9 (2015).

[24] J. Colgan, D. P. Kilcrease, N. H. Magee, Jr., G. S. J. Armstrong, J. Abdallah, Jr., M. E. Sherrill, C. J. Fontes, H. L. Zhang, and P. Hakel, High Energy Density Phys. 9, 369 (2013).

[25] J. Colgan, D. P. Kilcrease, N. H. Magee, Jr., J. Abdallah, Jr., M. E. Sherrill, C. J. Fontes, P. Hakel, and H. L. Zhang, High Energy Density Phys. 14, 33 (2015).

[26] R. E. H. Clark, J. Abdallah Jr, and J. B. Mann, Ap. J. 381, 597 (1991); J. Abdallah Jr, H. L. Zhang, C. J. Fontes, D.P. Kilcrease, and B. J. Archer, J. Quant. Spectrosc. Rad. Trans. 71, 107 (2001).

[27] P. Hakel and D. P. Kilcrease, 14th Topical Conference on Atomic Processes in Plasmas, Eds: J. S. Cohen, S. Mazevet, and D. P. Kilcrease, (New York: AIP), pp 190-202 (2004).

[28] R. D. Cowan, J. Opt. Soc. Am. 58, 924 (1968).

[29] S. Turck-Chièze, M. Le Pennec, J. E. Ducret, J. Colgan, D. P. Kilcrease, C. J. Fontes, N. Magee, F. Gilleron, and J. C. Pain, Astrophys. J. 823, 78 (2016).

[30] K. Nishihara et al., Phys. Plasmas 15, 056708 (2008).

[31] C. J. Fontes, J. Colgan, and J. Abdallah, Jr., in Modern Methods in CollisionalRadiative Modeling of Plasmas (Springer, Switzerland, Editor: Yu. Ralchenko, 2016). 